\title{
Pulpotomía con biodentine comparado con formocresol en pacientes con dentición primaria.
}

\section{Biodentine versus formocresol for pulpotomy in primary teeth.}

\author{
Javiera De Solminihac ${ }^{1,2}$, Sofía Pizarro ${ }^{1,2}$, Andrea Cárdenas ${ }^{1,2^{*}}$
}

1. Escuela de Odontología, Facultad de Medicina, Pontificia Universidad Católica de Chile, Santiago, Chile

2. Proyecto Epistemonikos, Santiago, Chile

* Correspondencia Autor: Andrea Cárdenas | Dirección: Centro Evidencia UC, Pontificia Universidad Católica de Chile, Diagonal Paraguay 476, Santiago, Chile | E-mail: cardenasandrea77@ gmail.com

\section{RESUMEN}

Introducción: En dentición primaria, la pulpotomía con formocresol ha sido convencionalmente utilizada para el tratamiento de caries cercanas a la pulpa. Sin embargo, la seguridad de este material ha sido cuestionada. Por otro lado, el biodentine ha sido propuesto como un biomaterial sustituto bioactivo de la dentina, pero no está clara su efectividad. Métodos: Realizamos una búsqueda en Epistemonikos, la mayor base de datos de revisiones sistemáticas en salud, la cual es mantenida mediante el cribado de múltiples fuentes de información, incluyendo MEDLINE, EMBASE, Cochrane, entre otras. Extrajimos los datos desde las revisiones identificadas, analizamos los datos de los estudios primarios, realizamos un metanálisis y una tabla GRADE para el resumen de los resultados. Resultados y conclusiones: Identificamos tres revisiones sistemáticas que en conjunto incluyeron dos estudios primarios, ambos correspondientes a ensayos aleatorizados. Concluimos que no se puede establecer con claridad si biodentine comparado con formocresol aumenta el éxito clínico y el éxito radiográfico en pulpotomía en pacientes con dentición primaria, debido a que la certeza de la evidencia existente ha sido evaluada como muy baja.

PALABRAS CLAVE

Pulpotomía terapéutica; Formocresol; Biodentine; Epistemonikos, GRADE.

\section{ABSTRACT}

Introduction: Pulpotomy with formocresol is the main treatment protocol following carious pulp exposure in primary teeth, but many concerns have been raised regarding its safety. Biodentine has been proposed as a bioactive dentine substitute, but there is still uncertainty regarding its effectiveness. Methods: We searched in Epistemonikos, the largest database of systematic reviews in health, which is maintained by screening multiple information sources, including MEDLINE, EMBASE, Cochrane, among others. We extracted data from the systematic reviews, reanalyzed data of primary studies, conducted a meta-analysis and generated a summary of findings table using the GRADE approach. Results and conclusions: We identified three systematic reviews including two studies overall, of which both were randomized trials. We are uncertain whether biodentine compared to formocresol for pulpotomy improves clinical and radiographic success, as the certainty of the evidence has been assessed as very low.

Int. J. Inter. Dent Vol. 13(3); 212-216, 2020. 


\section{PROBLEMA}

En dentición primaria, existen distintas alternativas de tratamiento para la caries dental profunda bajo el enfoque mínimamente invasivo. En casos donde la caries se encuentra cercana a la pulpa y no se observa sintomatología, el tratamiento indicado corresponde a la pulpotomía.

La pulpotomía corresponde a un procedimiento cuyo fin es preservar la pulpa radicular, evitar dolor e inflamación y mantener el diente primario en boca, hasta su recambio fisiológico. El material de elección para este procedimiento durante mucho tiempo ha sido el formocresol, con un alto porcentaje de éxito, cuya acción consiste en fijar el tejido pulpar radicular debido a su capacidad momificante ${ }^{[1]}$. Sin embargo, se ha planteado que sus niveles de toxicidad ${ }^{[2]}$, carcinogenicidad ${ }^{[2]}$, mutagenicidad ${ }^{[3]}$, y la dificultad para controlar el grado de penetración, perjudicarían el pronóstico de los pacientes.

Actualmente el biodentine se ha propuesto como una alternativa a este biomaterial, ya que actúa como sustituto bioactivo de la dentina. Se ha estudiado que este material podría convertirse en potencial sustituto del formocresol, sin embargo existe poca claridad sobre su efectividad ${ }^{[4]}$.

\section{MÉTODOS}

Realizamos una búsqueda en Epistemonikos, la mayor base de datos de revisiones sistemáticas en salud, la cual es mantenida mediante búsquedas en múltiples fuentes de información, incluyendo MEDLINE, EMBASE, Cochrane, entre otras. Extrajimos los datos desde las revisiones identificadas y analizamos los datos de los estudios primarios. Con esta información, generamos un resumen estructurado denominado FRISBEE (Friendly Summaries of Body of Evidence using Epistemonikos), siguiendo un formato preestablecido, que incluye mensajes clave, un resumen del conjunto de evidencia (presentado como matriz de evidencia en Epistemonikos), metanálisis del total de los estudios cuando sea posible, una tabla de resumen de resultados con el método GRADE y una sección de otras consideraciones para la toma de decisión

\section{Mensajes clave}

- No es posible establecer con claridad si biodentine comparado con formocresol aumenta el éxito clínico y el éxito radiográfico en pulpotomía en pacientes con dentición primaria, debido a que la certeza de la evidencia existente ha sido evaluada como muy baja.
Acerca del conjunto de evidencia para esta pregunta

Cuál es la evidencia
Véase matriz de evidencia
en Epistemonikos más
abajo.

Encontramos tres revisiones sistemáticas ${ }^{[2]}$, [5], [6] que incluyeron dos estudios primarios reportados en tres referencias ${ }^{[7], ~[8], ~[9], ~ t o d o s ~}$ correspondientes a ensayos aleatorizados.

\begin{tabular}{ll}
\hline & Todos los ensayos incluyeron \\
& pacientes con dentición primaria, con \\
& caries dentinaria profunda y necesidad \\
Qué tipo de pacientes & de pulpotomía terapéutica.
\end{tabular}

incluyeron los estudios*

Un ensayo incluyó 37 pacientes, en un rango de cuatro a ocho años ${ }^{[7]}$. El otro ensayo incluyó 38 pacientes, con una edad promedio de 5,8 años ${ }^{[9]}$.

Todos los ensayos evaluaron el uso de biodentine comparado con el uso de formocresol para pulpotomías terapéuticas en dientes primarios ${ }^{[2]}$ [5], [6]

Un ensayo realizó irrigación con solución salina antes de la aplicación del material en el grupo de biodentine y en el grupo de formocresol se aplicó una motita de algodón empapada

Qué tipo de intervenciones incluyeron los estudios* con formocresol diluido, colocado en cuernos pulpares durante 5 minutos después de la pulpotomía, seguido de apósitos cemento de zinc-eugenol reforzado $^{[7]}$

Otro ensayo ${ }^{[8]}$ reportó que se realizó una restauración provisoria con cemento de zinc-eugenol reforzado y cemento de ionómero de vidrio. Todos los dientes incluidos fueron restaurados con coronas de metal preformadas como restauración definitiva.

Los ensayos reportaron múltiples desenlaces, los cuales fueron agrupados por las revisiones sistemáticas de la siguiente manera:

Qué tipo de desenlaces midieron

- éxito clínico

- éxito radiográfico

El seguimiento promedio de los ensayos fue de 12 meses con un rango que fluctúa entre tres y ocho meses.

* La información sobre los estudios primarios es extraída desde las revisiones sistemáticas identificadas, no directamente desde los estudios, a menos que se especifique lo contrario.

\section{RESUMEN DE LOS RESULTADOS:}

La información sobre los efectos de realizar pulpotomía con biodentine comparado con formocresol en pacientes con dentición primaria está basada en dos ensayos aleatorizados que incluyeron 142 dientes primarios con caries extensas.

Todos los ensayos midieron éxito clínico y éxito radiográfico (142 dientes en 75 pacientes $)^{[7,9]}$. Ninguna de las revisiones sistemáticas reportó los desenlaces pérdida prematura del diente y eventos adversos.

El resumen de los resultados es el siguiente:

- No es posible establecer con claridad si biodentine comparado con formocresol aumenta el éxito clínico en pulpotomía en pacientes con dentición primaria, debido a que la certeza de la evidencia existente ha sido evaluada como muy baja.

- No es posible establecer con claridad si biodentine comparado con formocresol aumenta el éxito radiográfico en pulpotomía en pacientes con dentición primaria, debido a que la certeza de la evidencia existente ha sido evaluada como muy baja.

- No se encontraron estudios que evaluaran el desenlace pérdida prematura del diente.

- No se encontraron estudios que evaluaran el desenlace eventos adversos. 


\begin{tabular}{|c|c|c|c|c|}
\hline $\begin{array}{l}\text { Pacientes } \\
\text { Intervención } \\
\text { Comparación }\end{array}$ & $\begin{array}{l}\text { Pacientes con de } \\
\text { Pulpotomía con } \\
\text { Pulpotomía con }\end{array}$ & $\begin{array}{l}\text { tición primaria qu } \\
\text { odentine } \\
\text { rmocresol }\end{array}$ & requieren pul & \\
\hline \multirow{3}{*}{ Desenlaces } & \multicolumn{2}{|c|}{ Efecto absoluto* } & \multirow{3}{*}{$\begin{array}{l}\text { Efecto relativo } \\
\text { (IC } 95 \% \text { ) }\end{array}$} & \multirow{3}{*}{$\begin{array}{l}\text { Certeza de la } \\
\text { evidencia } \\
\text { (GRADE) }\end{array}$} \\
\hline & $\begin{array}{c}\text { CON } \\
\text { formocresol }\end{array}$ & $\begin{array}{c}\text { CON } \\
\text { biodentine } \\
\end{array}$ & & \\
\hline & \multicolumn{2}{|c|}{ Diferencia: dientes por 1000} & & \\
\hline \multirow[b]{2}{*}{ Éxito clínico** } & 958 por 1000 & 1000 por 1000 & \multirow[b]{2}{*}{$\begin{array}{l}\text { RR } 1,06 \\
(0,88 \text { a } 1,29)\end{array}$} & \multirow[b]{2}{*}{ Muy baja } \\
\hline & \multicolumn{2}{|c|}{$\begin{array}{c}\text { Diferencia: } 57 \text { más } \\
\text { (Margen de error: } 115 \text { menos a } 278 \\
\text { más) }\end{array}$} & & \\
\hline \multirow[b]{2}{*}{$\begin{array}{l}\text { Éxito } \\
\text { radiográfico*** }\end{array}$} & 944 por 1000 & 1000 por 1000 & \multirow[b]{2}{*}{$\begin{array}{l}\text { RR } 1,06 \\
(0,87 \text { a } 1,31)\end{array}$} & \multirow[b]{2}{*}{$\begin{array}{l}\oplus \bigcirc \bigcirc \bigcirc^{1,2} \\
\text { Muy baja }\end{array}$} \\
\hline & \multicolumn{2}{|c|}{$\begin{array}{c}\text { Diferencia: } 57 \text { más } \\
\text { (Margen de error: } 123 \text { menos a } 293 \\
\text { más) }\end{array}$} & & \\
\hline $\begin{array}{l}\text { Pérdida dentaria } \\
\text { prematura }\end{array}$ & \multicolumn{2}{|c|}{$\begin{array}{l}\text { El desenlace pérdida prematura no } \\
\text { fue medido. }\end{array}$} & -- & -- \\
\hline $\begin{array}{l}\text { Eventos } \\
\text { adversos }\end{array}$ & \multicolumn{2}{|c|}{$\begin{array}{l}\text { El desenlace eventos adversos no } \\
\text { fue reportado. }\end{array}$} & -- & -- \\
\hline \multicolumn{5}{|c|}{$\begin{array}{l}\text { Margen de error: Intervalo de confianza del 95\% (IC 95\%). } \\
\text { RR: Riesgo relativo. } \\
\text { GRADE: Grados de evidencia del GRADE Working Group (ver más adelante). }\end{array}$} \\
\hline \multicolumn{5}{|c|}{$\begin{array}{l}\text { *Los riesgos CON formocresol están basados en los riesgos del grupo control en los estudios. El } \\
\text { riesgo CON biodentine (y su margen de error) está calculado a partir del efecto relativo (y su } \\
\text { margen de error). } \\
\text { ** El desenlace "éxito clínico" fue definido como ausencia de sensibilidad, dolor o inflamación, sin } \\
\text { sensibilidad a la percusión, sin absceso o fístula y sin movilidad dental. } \\
\text { *** El desenlace "éxito radiográfico" fue definido como ausencia de furcación y radiolucidez } \\
\text { periapical, ausencia de reabsorción de raíz interna o externa. }\end{array}$} \\
\hline \multicolumn{5}{|c|}{$\begin{array}{l}{ }^{1} \text { Se disminuyó un nivel de certeza de evidencia por riesgo de sesgo, ya que no estaba claro el } \\
\text { proceso ni el ocultamiento de la secuencia de aleatorización, enmascaramiento y reporte selectivo. }\end{array}$} \\
\hline \multicolumn{5}{|c|}{$\begin{array}{l}{ }^{2} \text { Se disminuyó dos niveles de certeza de evidencia por imprecisión, pues a cada extremo del } \\
\text { intervalo de confianza se tomarían decisiones opuestas y existe amplio rango entre ellas. }\end{array}$} \\
\hline
\end{tabular}

Siga el enlace para acceder a la versión interactiva de esta tabla (Interactive Summary of Findings - iSoF)

\begin{tabular}{|l|}
\hline Acerca de la certeza de la evidencia (GRADE)* \\
\hline$\oplus \oplus \oplus \oplus$ \\
Alta: La investigación entrega una muy buena indicación del efecto probable. La probabilidad de \\
que el efecto sea sustancialmente distinto† es baja. \\
$\oplus \oplus \oplus \bigcirc$ \\
Moderada: La investigación entrega una buena indicación del efecto probable. La probabilidad de \\
que el efecto sea sustancialmente distinto† es moderada. \\
$\oplus \oplus \bigcirc \bigcirc$ \\
Baja: La investigación entrega alguna indicación del efecto probable. Sin embargo, la probabilidad \\
de que el efecto sea sustancialmente distinto† es alta. \\
$\oplus \bigcirc \bigcirc \bigcirc$ \\
Muy baja: La investigación no entrega una estimación confiable del efecto probable. La \\
probabilidad de que el efecto sea sustancialmente distinto† es muy alta. \\
\hline *Esto es también denominado 'calidad de la evidencia' o 'confianza en los estimadores del efecto'. \\
$\dagger$ Sustancialmente distinto = una diferencia suficientemente grande como para afectar la decisión \\
\hline
\end{tabular}




\section{OTRAS CONSIDERACIONES PARA LA TOMA DE DECISIÓN}

\section{A quién se aplica y a quién no se aplica esta evidencia}

- Las conclusiones presentadas en este resumen aplican a pacientes sanos con dentición primaria que necesitan pulpotomía terapéutica en dientes con caries extensas.

\section{Sobre los desenlaces incluidos en este resumen}

- Los desenlaces seleccionados son aquellos considerados críticos para la toma de decisiones, de los cuáles sólo el éxito clínico y radiográfico fueron reportados por las revisiones sistemáticas identificadas.

- Ningún estudio reportó los desenlaces pérdida dentaria prematura y eventos adversos, los cuales también son desenlaces considerados críticos por los autores de este resumen.

- Es necesario abordar la durabilidad de las intervenciones a largo plazo, ya que se ha sugerido que algunos pacientes pueden presentar pérdida prematura, relacionado con el uso de formocresol.

\section{Balance daño/beneficio y certeza de la evidencia}

- Debido a que existe incertidumbre importante asociada a los efectos de la intervención, no es posible realizar un correcto análisis del balance entre daños y beneficios, por lo que otros aspectos deben ser considerados para la toma de decisiones.

\section{Consideraciones de recursos}

- Ninguna de las revisiones sistemáticas identificadas realizó una valoración del costo efectividad de las intervenciones. El formocresol se encuentra a través de múltiples marcas a la venta, con un valor inferior al de biodentine, lo cual hace que sea más asequible en comparación a este último.

- Considerando precio y evidencia (balance entre riesgos y beneficios), no corresponde analizarlo aún hasta que se pruebe su beneficio.

\section{Qué piensan los pacientes y sus tratantes}

- Enfrentados a la evidencia existente, la mayoría de los pacientes y tratantes deberían inclinarse al uso de formocresol por sobre el uso de biodentine para realizar pulpotomías terapéuticas en dientes primarios, ya que este ha sido convencionalmente el material de elección para este tratamiento.

- Sin embargo, sus características citotóxicas y mutagénicas, además del potencial cancerígeno que se ha estudiado, han hecho que los pacientes cuestionen el uso del formocresol en el último tiempo. La principal preocupación ha sido el formaldehído, componente del formocresol[10]. Sin embargo, es poco probable que el formocresol represente un riesgo para los niños si se usa en dosis y forma adecuadas para el procedimiento de pulpotomía vital[11].

- El biodentine es un material nuevo, por lo que puede generar dudas en los pacientes. Principalmente se relaciona con mejores propiedades físicas y biológicas; como mejor manipulación, tiempo de fraguado rápido, porosidad disminuida y síntesis temprana de dentina reparativa comparado con otros materiales, siendo muy beneficioso para el tratante, sin embargo, su precio limita la accesibilidad al producto ${ }^{[7]}$.

\section{Diferencias entre este resumen y otras fuentes}

- Las conclusiones de este resumen no coinciden con las de las revisiones sistemáticas identificadas, ya que concluyen que no existen diferencias significativas con respecto al éxito clínico y éxito radiográfico entre usar biodentine versus formocresol en pulpotomía en dentición primaria. Estas diferencias pueden deberse a que las revisiones sistemáticas no evaluaron la certeza de la evidencia de sus resultados.

- Según la guía clínica de la AAPD 2014 (American Academy of Pediatric Dentistry $)^{[10]}$, la guía clínica Minsal $2013^{[13]}$ y European Journal of Paediatric Dentistry $2019^{[14]}$, el formocresol se recomienda si es usado con criterio; y establecen que es un medicamento seguro, por lo que no hay razones científicas o toxicológicas para discontinuar su uso en odontología pediátrica. Esto hasta que exista una alternativa biológica y reparadora que tenga resultados superiores y reproducibles.

- Otras fuentes como la Sociedad Española de Odontopediatría 2019[15], guía clínica Minsal 2013 ${ }^{[13]}$, establecen que a pesar de ello, es un agente ampliamente cuestionado por sus potenciales efectos tóxicos, carcinogénicos e inmunológicos, sin embargo hasta el momento no hay conclusiones firmes que lo confirmen respecto a su utilización como agente pulpar. Se destaca la preocupación que existe en su uso, sabiendo que existen otras alternativas que son igualmente eficaces. ${ }^{[15]}$

- En contraparte, de acuerdo a la guía Scottish Dental Clinical Effectiveness Programme de 2018, se establece que el formocresol no debiera usarse, debido a la incertidumbre que existe respecto a su seguridad ${ }^{[16]}$

\section{¿Puede que cambie esta información en el futuro?}

- Es muy probable que las conclusiones de este resumen cambien a futuro, debido a la incertidumbre de la evidencia existente.

- Se identificaron dos revisiones sistemáticas en curso en PROSPERO International prospective register of systematic reviews que evalúan resultados clínicos y radiográficos de pacientes que requieren pulpotomías y/o recubrimiento pulpar directo comparando distintos biomateriales ${ }^{[17][18] .}$

\section{CÓMO REALIZAMOS ESTE RESUMEN}

Mediante métodos automatizados y colaborativos recopilamos toda la evidencia relevante para la pregunta de interés y la presentamos en una matriz de evidencia.

\section{Siga el enlace para acceder a la versión interactiva:}

Pulpotomía con biodentine comparado con formocresol para dientes primarios.

\section{NOTAS}

Si con posterioridad a la publicación de este resumen se publican nuevas revisiones sistemáticas sobre este tema, en la parte superior de la matriz se mostrará un aviso de "nueva evidencia".

Este artículo es parte del proyecto síntesis de evidencia de Epistemonikos. Se elabora con una metodología preestablecida, siguiendo rigurosos estándares metodológicos y proceso de revisión por pares interno. Cada uno de estos artículos corresponde a un resumen, denominado FRISBEE (Friendly Summary of Body of Evidence using Epistemonikos), cuyo principal objetivo es sintetizar el conjunto de evidencia de una pregunta específica, en un formato amigable a los profesionales clínicos. Sus principales recursos se basan en la matriz de evidencia de Epistemonikos y análisis de resultados usando metodología GRADE. Mayores detalles de los métodos para elaborar este FRISBEE están descritos aquí (http://dx.doi.org/10.5867/medwave.2014.06.5997)

La Fundación Epistemonikos es una organización que busca acercar la información a quienes toman decisiones en salud, mediante el uso de tecnologías. Su principal desarrollo es la base de datos Epistemonikos (www.epistemonikos.org).

\section{DECLARACIÓN DE CONFLICTOS DE INTERESES}

Los autores declaran no tener conflictos de intereses con la materia de este artículo.

\section{AGRADECIMIENTOS}

Este resumen de evidencia fue elaborado con el apoyo metodológico del Centro Evidencia UC, Facultad de Medicina, Pontificia Universidad Católica de Chile. 


\section{Bibliografía}

1. Vallejo E, Rosales J. Materiales dentales. En: Boj JR. Odontopediatría. La evolución del niño al adulto joven. Madrid: Ripano editorial médica; 2011. p.195-210. 2. Smaïl-Faugeron V, Glenny AM, Courson F, Durieux P, Muller-Bolla M, Fron Chabouis H. Pulp treatment for extensive decay in primary teeth. Cochrane Database Syst Rev. 2018 May 31;5(5):CD003220. doi: 10.1002/14651858.CD003220.pub3. PMID: 29852056; PMCID: PMC6494507.

3. Azabal M, Vega del Barrio J.M. Cementos en odontología (II): cementos de hidróxido de calcio. Cementos de óxido de cinc-eugenol. En: Vega del Barrio JM. Materiales en odontología. Fundamentos biológicos, clínicos, biofísicos y físicoquímicos. Madrid: Ediciones Avances; 1996. p. 387-419.

4. Hincapié Narváez S, Valerio Rodriguez AL. Biodentine: Un nuevo material en terapia pulpar / Biodentine: A New Material for Pulp Therapy. Univ Odontol [Internet]. 2015 Dec 30; 34(73):69-76. Available from: http://dx.doi.org/10.11144/Jave-

5. Shafaee $\mathrm{H}$, Alirezaie M, Rangrazi A, Bardideh E. Comparison of the success rate of a bioactive dentin substitute with those of other root restoration materials in pulpotomy of primary teeth: Systematic review and meta-analysis. J Am Dent Assoc. 2019 Aug;150(8):676-688. doi: 10.1016/j.adaj.2019.03.002. Epub 2019 Jun 13. PMID: 31202439

6. Bossù M, laculli F, Di Giorgio G, Salucci A, Polimeni A, Di Carlo S. Different Pulp Dressing Materials for the Pulpotomy of Primary Teeth: A Systematic Review of the Literature. J Clin Med. 2020 Mar 19;9(3):838. doi: 10.3390/jcm9030838. PMID: 32204501; PMCID: PMC7141304.

7. El Meligy OA, Allazzam S, Alamoudi NM. Comparison between biodentine and formocresol for pulpotomy of primary teeth: A randomized clinical trial. Quintessence Int. 2016;47(7):571-80. doi: 10.3290/j.qi.a36095. PMID: 27175451.

8. El Meligy OAES, Alamoudi NM, Allazzam SM, El-Housseiny AAM Biodentine<sup $>\mathrm{TM}</$ sup $>$ versus formocresol pulpotomy technique in primary molars: a 12-month randomized controlled clinical trial. BMC Oral Health. 2019 Jan 7;19(1):3. doi: 10.1186/s12903-018-0702-4. PMID: 30612569; PMCID: PMC6322259.

9. Juneja P, Kulkarni S. Clinical and radiographic comparison of biodentine, minera trioxide aggregate and formocresol as pulpotomy agents in primary molars. Eur Arch Paediatr Dent. 2017 Aug;18(4):271-278. doi: 10.1007/s40368-017-0299-3. Epub 2017 Aug 5. PMID: 28780718.

10. Peng, L., Ye, L., Guo, X., Tan, H., Zhou, X., Wang, C., \& Li, R. (2007). Evaluation of formocresol versus ferric sulphate primary molar pulpotomy: a systematic review and meta-analysis. International Endodontic Journal, 40(10), 751-757. doi:10.1111/ j.1365-2591.2007.01288.x

11. Marghalani, A. A., Omar, S., \& Chen, J.-W. (2014). Clinical and radiographic success of mineral trioxide aggregate compared with formocresol as a pulpotomy treatment in primary molars. The Journal of the American Dental Association, 145(7), 714-721. doi:10.14219/jada.2014.36

12. Kratunova E, Silva D. Pulp therapy for primary and immature permanent teeth: An overview. Gen Dent. 2018;66(6):30-8. Available from: https://www.aapd.org/ media/Policies Guidelines/BP PulpTherapy.pdf

13. Ministerio de Salud. "Guía Clínica Salud Oral integral para niños y niñas de 6 años". [Internet]. 2013. Available from: http://www.bibliotecaminsal.cl/wp/wpcontent/uploads/2016/04/Salud-Oral-Integral-niños-y-niñas-6-años.pdf

14. Olczak-Kowalczyk D, Samul M, Góra J, Gozdowski D, Turska-Szybka A. Ferric Sulfate and Formocresol pulpotomies in paediatric dental practice. A prospectiveretrospective study. Eur J Paediatr Dent. 2019;20(1):27-32.

15. Sociedad Española de Odontopediatría PROTOCOLO PARA LOS TRATAMIENTOS PULPARES EN DENTICIÓN TEMPORAL [Internet]. 2019 Available from: https://www.odontologiapediatrica.com/wp-content/uploads/2019/02/ SEOP-Protocolo-PULPA-Actualizado-31012019.pdf

16. NICE. Prevention and Management of Dental Caries in Children guidance. Scottish Dent Clin Eff Program Dundee [Internet]. 2018; Available from: http://www. sdcep.org.uk/wp-content/uploads/2018/05/SDCEP-Prevention-and-Managementof-Dental-Caries-in-Children-2nd-Edition.pdf

17. Venkateshbabu Nagendrababu, Shaju Jacob Pulikkotil, Sajesh K Veettil, Paul Dummer. Comparing the clinical and radiographic outcomes of pulpotomies in primary molars using bioactive endodontic materials or formocresol as the pulp capping material - a systematic review and meta-analysis with trial sequential analysis of randomized controlled trials. PROSPERO 2018 CRD42018089064 Available from: https://www.crd.york.ac.uk/prospero/display_record.php?ID=CRD42018089064

18. Athanasios Fasoulas, Georgios Keratiotis, Maarten Augustus Meire, Nikolaos Pandis, Loukia Spineli. Comparative efficacy of materials used in patients undergoing pulpotomy or direct pulp capping: A systematic review with network meta-analysis. PROSPERO 2020 CRD42020127239 Available from: https://www. crd.york.ac.uk/prospero/display record.php?ID=CRD42020127239 